\title{
2020: Archivos en tiempos de la COVID-19
}

\author{
2020: Archivos in Times of COVID-19
}

\author{
Laura Fontcuberta ${ }^{1}$ \\ Jordi Delclós ${ }^{1}$ \\ Guillermo García' \\ José Miguel Martínez ${ }^{1}$ \\ José M. Ramada' \\ María del Mar Seguí \\ Elena Ronda ${ }^{\top}$
}

${ }^{1}$ Archivos de Prevención de Riesgos Laborales, Barcelona, España.

Fechas · Dates

Recibido: 2021.01.10

Publicado: 2021.01.21
Correspondencia · Corresponding Author

Elena Ronda

Universidad de Alicante

elena.ronda@ua.es 
En el momento de la redacción de esta nota editorial, seguimos mirando con preocupación y temor la evolución de la situación de la infección originada por el SARSCoV-2, pero también con esperanza por el comienzo de la vacunación. Los retos que la pandemia ha representado y representa para la salud laboral son inmensos: cambios en las condiciones de trabajo, afectación del mercado laboral, aparición de nuevas formas de trabajo, el papel de los servicios de prevención en la lucha contra la transmisión de la enfermedad, así como en la detección y control de trabajadores infectados y sus contactos, el medio laboral como lugar de transmisión de la enfermedad o la identificación de trabajadores especialmente sensibles y vulnerables, son algunos de los ejemplos que podríamos citar ${ }^{(1-4)}$.

Desde el inicio de la pandemia por COVID-19, se ha producido también la mayor publicación de artículos científicos conocida con la finalidad de aclarar las principales incógnitas acerca de las vías de transmisión, la sintomatología y curso clínico, las pruebas de detección y los diferentes abordajes preventivos y terapéuticos de esta nueva enfermedad ${ }^{(5)}$. Archivos también ha estado ahí. En el número publicado en marzo de 2020 se incluyeron ya dos editoriales ${ }^{(6,7)}$. Una de ellas, referente a la salud de los trabajadores y COVID(6), alcanzó más de 4500 descargas durante los tres primeros meses, sobrepasando en la actualidad las 7000. También se ha habilitado en la página web un enlace a todos los contenidos publicados relacionados con la COVID-19 (https://archivosdeprevencion.eu/index.php/aprl/catalog/ category/covid19) para facilitar su acceso de forma directa.

Centrándonos ya en la propia gestión de la revista, hay que mencionar que, después del salto cualitativo que representó la adopción de la herramienta de gestión editorial de las revistas Open Journal Systems (OJS)(8) (a la que se puede acceder desde este enlace http://archivosdeprevencion.eu/index.php/aprl/login, y que ya tiene disponibles todos los números de la revista), hemos dado otro para aumentar el acceso y llegar a nuevo público a través de las redes sociales. Así, desde 2020, la revista ya está activa en Facebook y Twitter. Animamos a uniros a los seguidores de Archivos en APRL en Facebook y APRL en Twitter, para estar informados al momento de cualquier novedad.
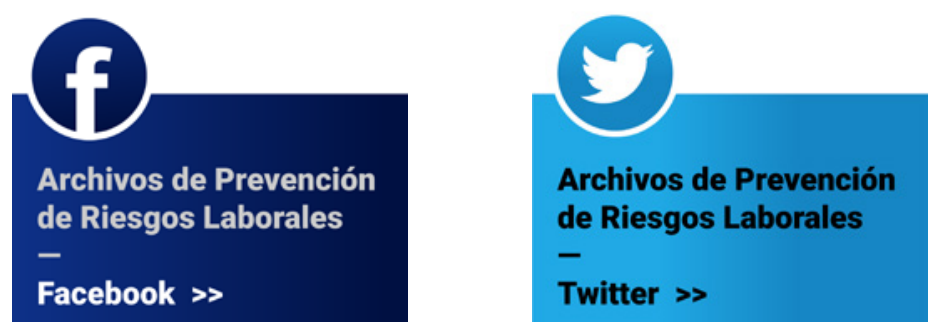

En el empeño de visualizar la valiosa labor científica de los autores que publican sus trabajos en Archivos, nos esforzamos para lograr su inclusión en nuevas bases de datos, plataformas y directorios. Como noticia relevante, tenemos que celebrar que la revista ya está incluida, además de en PubMed, en SCOPUS, lo que la consolida como referente de transmisión de conocimiento científico en lo que respecta a la salud laboral en lengua castellana. Además, seguimos con el proceso de indización en Web of Science, DOAJ, LATINDEX 2.0 y ERIH PLUS. 


\section{Gestión de manuscritos: número y tipos de trabajos}

Empezamos este resumen revisando los manuscritos gestionados durante el último año en relación con los de los dos años anteriores. Se puede visualizar en la Tabla 1 el incremento de manuscritos gestionados respecto a los dos años anteriores, sobre todo respecto a 2019. Como siempre, animamos a nuestros lectores a enviar sus trabajos a revisión en el formato (original, original breve, estudio de casos, nota técnica, etc.) que se adapte mejor a sus intereses.

Tabla 1: Manuscritos ${ }^{a}$ gestionados en Archivos de Prevención de Riesgos Laborales (2018 a 2020, volúmenes 21,22 y 23 )

\begin{tabular}{lccc}
\hline & $\mathbf{2 0 1 8}$ & $\mathbf{2 0 1 9}$ & $\mathbf{2 0 2 0}$ \\
\hline Aceptados & 28 & 22 & 25 \\
Rechazados & 22 & 14 & 21 \\
Abandonos & 2 & 4 & 0 \\
En proceso & 5 & 6 & 12 \\
Total & 57 & 46 & 58 \\
\hline
\end{tabular}

ancluye editoriales, originales, originales breves, artículos especiales, revisiones, artículos de opinión, estudios de casos, notas técnicas y cartas al director hasta el 2 de diciembre de 2020.

bEn el momento de elaborarse las Notas Editoriales de los correspondientes años.

En la Tabla 2 se presenta la distribución por tipo de trabajo publicado, destacando el aumento de originales publicados $(n=11)$. Este aumento es muy significativo porque es la primera vez que se logra pasar la decena de originales publicados.

Tabla 2: Tipos de artículos ${ }^{a}$ publicados en Archivos de Prevención de Riesgos Laborales (2018 a 2020 , volúmenes 21,22 y 23).

\begin{tabular}{lccc}
\hline & $\mathbf{2 0 1 8}$ & $\mathbf{2 0 1 9}$ & $\mathbf{2 0 2 0}$ \\
\hline Editoriales & 5 & 4 & 6 \\
Notas Editoriales & 1 & 1 & 1 \\
Originales $^{b}$ & 6 & 7 & 11 \\
Revisiones & 2 & 0 & 3 \\
Estudios de casos & 0 & 2 & 2 \\
Notas Técnicas & 0 & 2 & 0 \\
Cartas a la directora $^{\circ}$ & 3 & 0 & 2 \\
Otros $^{\circ}$ & 11 & 4 & 3 \\
\hline
\end{tabular}

Excluye secciones informativas (Sociedades, Noticias, Archivos Selección, Archivos Evidencia, Archivos

Formación).

Incluye Originales Breves.

-Otros: Comunicaciones de la Diada de la @SCSL, Artículos Especiales, Artículos de Opinión, eto 
Las medianas de los tres indicadores de los tiempos de gestión (recepción-aceptación, aceptación-publicación, y recepción-publicación) se han visto incrementados respecto a los años anteriores, al igual que los de recepción a aceptación (Tabla 3). La demora en el primer indicador creemos que podría atribuirse a la sobrecarga laboral provocada por la situación de pandemia y que ha afectado tanto a autores como a evaluadores y editores.

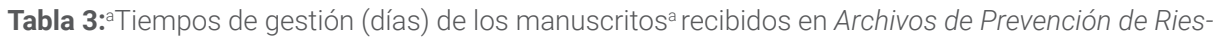
gos Laborales (2018 a 2020 volúmenes 21, 22 y 23)

\begin{tabular}{lcccccc}
\hline & \multicolumn{2}{c}{$\mathbf{2 0 1 8}$} & \multicolumn{2}{c}{$\mathbf{2 0 1 9}$} & \multicolumn{2}{c}{$\mathbf{2 0 2 0}$} \\
\hline & Mediana & Media & Mediana & Media & Mediana & Media \\
\hline Recepción-aceptación & 102 & 96 & 102 & 96 & 140 & 156 \\
Aceptación-publicación & 52 & 68 & 52 & 68 & 71 & 65 \\
Recepción-publicación & 146 & 165 & 146 & 165 & 233 & 158 \\
\hline
\end{tabular}

a|ncluye sólo originales, originales breves, revisiones y estudios de casos.

\section{Autorías y contenidos}

Respecto a la Tabla 4, relativa a las características de los autores según género y procedencia geográfica, debe destacarse que se mantiene el ligero incremento de diversidad geográfica en cuanto a procedencia que ya habíamos detectado en la anterior nota editorial, y también una clara presencia de mujeres como primeras autoras, por el otro.

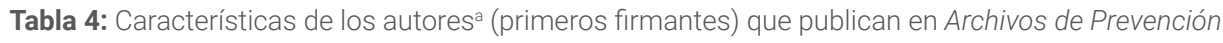
de Riesgos Laborales (2018 a 2020, volúmenes 21, 22 y 23).

2018

2019

2020

\begin{tabular}{llll}
\hline Género & & & \\
\hline Hombres & 12 & 9 & 9 \\
Mujeres & 14 & 8 & 16
\end{tabular}

\section{Procedencia}

Andalucía

Aragón

Asturias

Cantabria

Castilla y León

Cataluña

c. Valenciana

Extremadura

Galicia

Islas Baleares

Islas Canarias

La Rioja

Madrid

Navarra

País Vasco

Otros países 


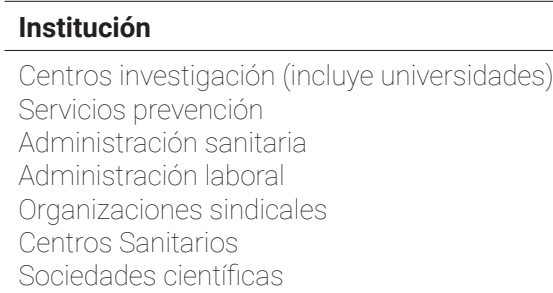

Excluye secciones fijas (Noticias, Archivos Selección, Archivos Evidencia, Archivos Formación).

En cuanto a los contenidos tratados (reflejados en la Tabla 5), como viene siendo habitual, son los relativos a políticas y estrategias para la prevención y problemas de salud relacionados con el trabajo los más frecuentes.

Tabla 5: Artículos publicados en Archivos de Prevención de Riesgos Laborales (volumen 23, año 2020) según contenidos .

\begin{tabular}{lccccc}
\hline & $\mathbf{N}^{\mathbf{1}}$ & $\mathbf{N}^{\mathbf{0}} \mathbf{2}$ & $\mathbf{N}^{\mathbf{0}}$ & $\mathbf{N}^{\mathbf{0}} \mathbf{4}$ & Total $\mathbf{2 0 2 0}$ \\
\hline Políticas y estrategias para la prevención & 2 & 5 & 2 & 2 & 11 \\
Historia de la salud laboral & 1 & & & & 1 \\
Evaluación de intervenciones & & & & & \\
Vigilancia de la salud & & 1 & 1 & 1 & 3 \\
Problemas de salud relacionados con el trabajo & 1 & 3 & 2 & 2 & 8 \\
Evaluación de riesgos & 1 & & 1 & & 2 \\
\hline
\end{tabular}

Excluye secciones fijas (Noticias, Archivos Selección, Archivos Evidencia y Archivos Formación), notas editoriales y comunicaciones de la Diada de la @SCSL.

Antes de terminar, no podía faltar nuestro agradecimiento a los indispensables que con su esfuerzo hacen posible cada número publicado: los distintos comités, evaluadores externos -que como siempre son reconocidos públicamente en el listado que aparece a continuación de esta nota-, y a los coordinadores de Archivos Evidencia y Archivos Selección -Guillermo García, Consol Serra y Mar Seguí- y de las noticias, Amàlia Valls. Queremos también mencionar, en especial, a Silvia Royo y Águeda Giráldez, que hasta este 2020 y a lo largo de estos años han colaborado con Archivos, proporcionando contenidos en el primer caso, relativos a noticias y congresos, y elaborando la sección Archivos Formación, en el segundo. Muchas gracias a ambas por vuestra gran labor. Un agradecimiento especial también a Motu Estudio por su disponibilidad y ayuda en la gestión de la plataforma y en la publicación de cada número. El apoyo de la Junta de la Associació Catalana de Salut Laboral (@SCSL) así como de sus socios es inestimable para la existencia de Archivos.

Y, claro está, a nuestros autores y lectores, el auténtico estímulo para seguir con la tarea del día a día.

A todos ellos nuestros mejores deseos para el próximo año. 


\section{Bibliografía}

1. Burdorf A, Porru F, Rugulies R. The COVID-19 (Coronavirus) pandemic: consequences for occupational health. Scand J Work Environ Health 2020(3):229-30. doi: 10.5271/sjweh.3893

2. Zhang M. Estimation of differential occupational risk of COVID-19 by comparing risk factors with case data by occupational group. Am. J. Ind. Med 2021;64(1):3947. doi: https://doi.org/10.1002/ajim.23199

3. Sasaki N, Kuroda R, Tsuno K, Kawakami N. The deterioration of mental health among healthcare workers during the COVID-19 outbreak: A population-based cohort study of workers in Japan. Scand J Work Environ Health. 2020;46(6):639644. doi:10.5271/sjweh.3922.

4. The Lancet. The plight of essential workers during the COVID-19 pandemic. Lancet (London, England) 2020;395(10237):1587.

5. Leiva-Cepas F, Romero-Rodríguez E, Sevillano MB. Las revistas científicas ante la pandemia por COVID-19. Semergen 2020;46:1-2.

6. Benavides FG. La salud de los trabajadores y la COVID-19. Arch Prev Riesgos Labor 2020;23(2):154-58.

7. Purtí $E$, Inglés J, de la Peña L, Rodríguez MC, Puiggenè $M$, Bernad MC, Calvet S, et al. La Asociación Catalana de Salud Laboral (@SCSL) ante el gran reto de la COVID-19. Arch Prev Riesgos Labor 2020;23(2): 146-53.

8. Fontcuberta L, Delclós J, García G, Martínez JM, Ramada JM, Ronda E. Archivos 2019: Bienvenido OJS, apuesta decidida por la calidad y la visibilidad editorial. Arch Prev Riesgos Labor 2020;23(1):14-1. 


\section{Evaluadores externos para Archivos de Prevención de Riesgos Laborales (2020)}

\begin{tabular}{|c|c|c|c|}
\hline Nombre & Apellidos & País & Afiliación \\
\hline Luis Fidel & Abregú Tueros & PE & Universidad Nacional Agraria de la Selva, Perú \\
\hline Sara & Alguacil & ES & Mc Mutual \\
\hline Patxi & Alija & ES & Adimen Investigación \\
\hline Alfonso & Apellaniz González & ES & Facultad de Medicina y Odontología, EHU-UPV \\
\hline Amaia & Ayala Garcia & ES & CISAL-UPF \\
\hline Fernando & Benavides & ES & CISAL-UPF \\
\hline Shyrle & Berrio García & $\mathrm{CO}$ & Pontificia Universidad Javeriana, Colombia \\
\hline Mar & Blasco & ES & Universidad Miguel Hernández \\
\hline Pere & Boix & ES & i+3 consultores \\
\hline Francisco & Brocal & ES & Universidad de Alicante \\
\hline Santiago & Calvet & ES & Asociación Española de Higiene Industrial (AEHI) \\
\hline Beatriz & Calvo-Cerrada & ES & CISAL-UPF \\
\hline Javier & Campos Serna & ES & Centro de salud Vila Joyosa \\
\hline Natalia & Cantó Sancho & ES & Universidad de Alicante \\
\hline Pablo & Chico Sánchez & ES & Hospital General de Alicante \\
\hline Cecilia & Cornelio & AR & Ministerio de Trabajo, Argentina \\
\hline Julia & del Prado Vinuesa & ES & Centre d'Investigació en Salut Laboral \\
\hline Jordi & Delclós & US & University of Texas, Estados Unidos \\
\hline Vicenta & Escribá & ES & $\begin{array}{l}\text { Conselleria de Sanidad de la Generalidad } \\
\text { Valenciana }\end{array}$ \\
\hline Iván & Fernández Suárez & ES & Universidad Internacional de La Rioja \\
\hline Vega & Garcia Lopez & ES & Instituto de Salud Pública y Laboral de Navarra \\
\hline Patricia & Garcia Shimizu & ES & Hospital de Villajoyosa \\
\hline Italo Nicolás & Girao Popolizio & ES & MC Mutual \\
\hline Juan & Gómez Salgado & ES & Universidad de Huelva \\
\hline Joan & Ingles Torruella & ES & Societat catalana de Salud Laboral \\
\hline Julio & Lavarello-Salinas & ES & Universitat de Valencia \\
\hline Juan Carlos & López & ES & MC Mutual \\
\hline Diego jose & López Rodríguez & ES & Master en PRL \\
\hline Francisco & Marqués & ES & $\begin{array}{l}\text { Instituo Nacional de Seguridad y Salud en el } \\
\text { Trabajo }\end{array}$ \\
\hline Anna & Martí Margarit & ES & Cualtis \\
\hline Juan Carlos & Martín & ES & Universitat Internacional de Catalunya \\
\hline José Miguel & Martínez & ES & MC Mutual \\
\hline Antonio & Merelles & ES & Universidad de Valencia \\
\hline María José & Molina Torres & ES & INVASSAT Alicante \\
\hline
\end{tabular}




\begin{tabular}{|c|c|c|c|}
\hline Nombre & Apellidos & País & Afiliación \\
\hline Albert & Navarro & ES & Universitat Autònoma de Barcelona \\
\hline Andreu & Nolasco Bonmati & ES & Universidad de Alicante \\
\hline Rosa Maria & Orriols Ramos & ES & Hospital Universitari de Bellvitge \\
\hline Claudia & Palma Vasquez & ES & CISAL-UPF \\
\hline José Luis & Pascual Arribas & ES & $\begin{array}{l}\text { Centre de Seguretat i Salut Laboral de Barcelona } \\
\text { de la Generalitat de Catalunya. }\end{array}$ \\
\hline Raúl & Payá Castiblanque & ES & Universidad de Valencia \\
\hline Pilar & Peña García & ES & $\begin{array}{l}\text { Servei de Prevenció. Consorci Corporació } \\
\text { Sanitària Parc Taulí de Sabadell }\end{array}$ \\
\hline Àngel & Plans & ES & ICO Institut Català d'Oncologia \\
\hline Maria Cruz & Rodríguez-Jareño & ES & Universitat de Girona \\
\hline Elena & Ronda & ES & Universidad de Alicante \\
\hline Carlos & Ruíz Frutos & ES & Universidad de Huelva \\
\hline Maite & Sampere & ES & MC Mutual \\
\hline María del Mar & Sánchez Brau & ES & Alicante Visión S.L. \\
\hline Alberto & Sánchez Lite & ES & Universidad de Valladolid \\
\hline Ana & Tauste Francés & ES & AITEX Instituto tecnológico textil \\
\hline Fermín & Torrano Montalvo & ES & UNIR \\
\hline Mònica & Ubalde-Lopez & ES & IsGlobal \\
\hline Mireia & Utzet Sadurni & ES & CISAL-UPF \\
\hline Sergio & Vargas-Prada & UK & $\begin{array}{l}\text { Institute of Health \& Wellbeing. University of } \\
\text { Glasgow. Escocia }\end{array}$ \\
\hline Teofila & Vicente & ES & Servicio de Prevención. Grupo Correos. Valencia \\
\hline Kevin & Vigil Vega & ES & CISAL-UPF \\
\hline Vicent & Villanueva & ES & $\begin{array}{l}\text { Dirección General de Salud Pública. Conselleria } \\
\text { de Sanitat, Generalitat Valenciana. }\end{array}$ \\
\hline Guanlan & Zhao & $\mathrm{CHN}$ & Universidad de Alicante \\
\hline
\end{tabular}

\section{¡Con nuestro reconocimiento y gratitud!}

